\title{
In vitro compressive strength and edge stability testing of directly repaired glass-ionomer cements
}

\author{
Jing Zhang ${ }^{1} \cdot$ Peter Braun ${ }^{2} \cdot$ Avijit Banerjee $^{1,3}$ (I) \\ Received: 15 August 2019 / Accepted: 2 December 2019 / Published online: 17 December 2019 \\ (C) The Author(s) 2019
}

\begin{abstract}
Objective To study the repair potential of seven commercial glass-ionomer cements (GICs) using an in vitro edge compression test model.

Materials and methods A total of 448 normal and 192 repaired cylindrical specimens $(6 \times 4 \mathrm{~mm})$ were produced from 6 GICs and one resin-modified GIC. Repaired samples consisted of a base aged for 1 month before repaired by an overlying layer. All samples were matured for 1 day, 1 week, 1 month or 3 months before compression, and edge tests were performed respectively on the whole surface (compressive strength, CS) or on the edge (edge stability, ES) using a universal testing machine.

Results For normal specimens, Ketac Universal (KU) illustrated a significantly higher CS than other groups at all time points $(p<0.001)$. ES of KU was weaker than EQUIA Forte (EQF), FIX (Fuji IX) and RSC (Riva Self Cure) after 1 day, increasing after 1 week. Repaired specimens showed CS comparable to normal specimens $(p>0.05)$. Repaired KU significantly improved CS compared to repaired EQF and Fuji II (FII) after 1 day. No statistical difference was found in ES among these groups $(p>0.05)$. Conclusions KU provided the fastest maturation and greatest CS and ES in both normal and repair models after short-term ageing. Repair of GICs could potentially be achieved directly onto the fractured substrate and the subsequent improved mechanical performance could be maintained for at least 3 months.

Clinical relevance This study provides a potential alternative in-vitro method to assess GIC restoration failure as well as provide insight into the mechanisms of GIC restoration repair.
\end{abstract}

Keywords Glass-ionomer cement · GIC $\cdot$ Compressive strength $\cdot$ Edge stability test $\cdot$ In-vitro $\cdot$ Repair

\section{Introduction}

Glass-ionomer cement (GIC) is widely used as a biointeractive dental restorative material due to its aesthetic [1]

Electronic supplementary material The online version of this article (https://doi.org/10.1007/s00784-019-03170-x) contains supplementary material, which is available to authorized users.

Avijit Banerjee

avijit.banerjee@kcl.ac.uk

1 Centre for Oral Clinical \& Translation Sciences, Faculty of Dentistry, Oral \& Craniofacial Sciences, King's College London, Tower Wing, Guy's Dental Hospital, Great Maze Pond SE1 9RT UK, UK

2 Research \& Development Department, 3M Oral Care Solutions Division, Seefeld, Germany

3 Conservative \& MI Dentistry; Faculty of Dentistry, Oral \& Craniofacial Sciences, King's College London, Floor 25, Tower Wing, Guy's Dental Hospital, Great Maze Pond, King's Health Partners, London SE1 9RT, UK and anti-cariogenic properties, biocompatibility and direct adhesion to dental tissues [2]. The clinical use of restorative GICs includes Class I, II, III and V cavities in primary teeth as well as Class III and V cavities in permanent teeth [3]. Modern GICs have shown some clinical success in their use in posterior Class I load-bearing scenarios [4]. The major criticism of GIC is its weaker mechanical properties which limit its application in posterior load-bearing Class II restorations. A comparative study exhibited that mechanical properties, such as compressive strength, flexural strength and flexural elastic modulus, of GICs are significantly weaker than those of resin composites [5].

In clinical practice, bulk or margin fractures account for the predominant number of GIC tooth-restoration complex (TRC) failures [6-8]. In a recent systematic review, Ruengrungsom et al. [9] concluded that not only marginal fracture of occlusal restorations but also the loss of the proximal contact from marginal ridge chipping is also a significant concern for Class II restorations. Hence, the importance of introducing a 
specific edge test into the in vitro mechanical test methodology is emerging in assessing GICs and other restorative materials. An edge chipping test has been applied to dental restoration materials previously [10]. With the assistance of computer aided design and machining (CAD/CAM), Quinn et al. successfully reproduced the marginal chipping on glass ceramics, filled resin composites, zirconia and resin-based denture teeth by using an edge chipping machine $[10,11]$. A more recent report by Ilie [12] introduced a simplified edge testing method to evaluate GIC's edge toughness. The results suggest this method to be reliable and could be indicative regarding the tendency of GIC materials towards chipping.

On GIC TRC failure, Green et al. [13] outlined the '5Rs' management protocol which consists of Reviewing, Refurbishment, Resealing, Repair and Replacement of such restorations, according to the philosophy of minimally invasive (MI) dentistry. This protocol will reduce the risk of unnecessary total replacement of failed TRCs that may have a number of adverse effects including further irreversible damage of natural tooth structure, spiralling TRC failure, cusp fracture and difficulty in future re-restoration [14-16]. Using the 5Rs strategy, repairing directly on the existing failed GIC restoration can be considered a viable clinical option $[13,17]$. In past decades, there have been limited studies investigating the direct repair of GICs. An old in vitro study repaired GIC with a new GIC layer and found that shear bond strength at the interface reached at least $65 \%$ of the unrepaired material after $15 \mathrm{~min}, 24 \mathrm{~h}$ and 6 days [18]. In this study, specific preconditioning protocols were applied on the failed restoration surface prior to the subsequent repair. For example, phosphoric acid etching for $20 \mathrm{~s}$ or surface roughening followed by acid etching gave the best performance than other treatments such as maleic acid etching [18]. Similarly, Pearson et al. [19] showed that phosphoric acid etching provided suitable surface for micromechanical keying of fresh GIC materials. Jamaluddin and Pearson [20] compared the efficacy of etching by phosphoric acid and polyacrylic acid (PAA) and found that PAAtreated specimens showed flexural strength inferior to phosphoric acid, indicating a weaker repair. More recently, Maneenut et al. [21] and Welch et al. [22] reported studies on repairing of RMGICs and concluded that the repair was warranted when addition of resin-based adhesive was applied to a pre-treated surface. However, a common feature of these published studies is that separate clinical pre-treatment step was implemented to create a roughened surface. To the best of the authors' knowledge, the direct repair of contemporary GIC restorations has not been reported to include their edge stability in in vitro simulations.

Therefore, the aim of this study was twofold: to test the potential of a novel in vitro repair model in assessing the marginal fracture toughness of restorative materials, namely GICs and to investigate the mechanical performance of commercial GIC products using a conventional compression method and a novel edge compression testing model. The null hypothesis was that the GICs cannot be repaired using the repairing model.

\section{Materials and methods}

\section{Sample preparation}

Sample size was calculated using SPSSTM SamplePower ${ }^{\mathrm{TM}}$ software package 3.01 (IBM, USA). The assumptions were based on the mean compressive strength values from the pilot study. The significance (alpha) was set to 0.05 , number of categories was set to 7 and standard deviation was set to 40 . The calculation showed that eight specimens per group would yield a power of 0.97. A total of 448 standard specimens were prepared using a cylindrical mould with a diameter of $4 \mathrm{~mm}$ and a height of $6 \mathrm{~mm}$. Six commercial glass-ionomer cement materials (GICs) and one resin-modified glass-ionomer cement (RMGIC) were used: Ketac $\AA$ Universal Aplicap ${ }^{\circledR}$ (KU) (Neuss, 3M Germany), EQUIA Forte ${ }^{\circledR}$ (EQF) (Leuven, GC, Belgium), Fujiß II LC resin-modified GIC (FII) (Leuven, GC, Belgium), ChemFil Rock (CFR) (Konstanz, Dentsply Sirona, Germany), Fuji® IX GP Capsule (FIX) (Leuven, GC, Belgium), IonoStar Plus (ISP) (Cuxhaven, VOCO, Germany) and Riva Self Cure (RSC) (Victoria, SDI, Australia). The compositions of each material are summarized in Table 1. All materials were prepared following manufacturers' instructions and loaded into the standard cylindrical moulds, as shown in Fig. 1a, c. The moulds were clamped tightly by two glass slides on both sides. For FII RMGIC, light curing (calibrated, tip inner diameter $10 \mathrm{~mm}$, Elipar $^{\mathrm{TM}}$ DeepCure-S, 3 M Germany) was performed for $20 \mathrm{~s}$ on both sides with a light intensity of $1470 \mathrm{~mW} / \mathrm{cm}^{2}$ and wavelength of $430-480 \mathrm{~nm}$. Specimens were stored in a water bath (tap water) at $37{ }^{\circ} \mathrm{C}$ for $1 \mathrm{~h}$ before demoulding. Afterwards, specimens were transferred into glass jars filled with tap water and stored in the incubator (MIR-262, Sanyo, Japan) at $37^{\circ} \mathrm{C}$ for 1 day, 1 week, 1 month and 3 months.

A further 192 repaired specimens consisted of a coneshape GIC base and separate overlying repair material, as illustrated in Fig. 1b, d. For this test, two GICs and 1 RMGIC were used: KU, EQF and FII. The cone-shape bases were prepared by condensing the mixed material into customized cone-shaped moulds with a convergence angle of $2.86^{\circ}$. The moulds were clamped by two glass slides and stored in a water bath at $37{ }^{\circ} \mathrm{C}$ for $1 \mathrm{~h}$. For FII, light curing (Elipar ${ }^{\mathrm{TM}}$ DeepCure-S, $3 \mathrm{M}$, Germany) was performed for $20 \mathrm{~s}$ on both sides as described above. Afterwards, the cone-shape bases were demoulded and transferred into glass jars filled with tap water and stored in the incubator (MIR-262, Sanyo, Japan) at $37^{\circ} \mathrm{C}$ for 1 month. The aged bases were blotted with tissue paper to remove surface standing moisture and then 
Table 1 Tested materials and their chemical compositions

\begin{tabular}{|c|c|c|c|c|}
\hline Product name & Manufacturer & Compositions & Shade & Lot number \\
\hline \multirow[t]{2}{*}{ Ketac Universal (KU) } & \multirow[t]{2}{*}{$3 \mathrm{M}$} & Powder: Oxide glass & A2 & 70809A \\
\hline & & Liquid: Copolymer of acrylic acid-maleic acid, Tartaric acid, water & A4 & 3253922 \\
\hline \multirow[t]{2}{*}{ Equia Forte (EQF) } & \multirow[t]{2}{*}{$\mathrm{GC}$} & Powder: Fluoro-aluminosilicate glass & $\mathrm{A} 2$ & 170117A \\
\hline & & Liquid: Polyalkenoic acid, tartaric acid, water & B3 & $170614 \mathrm{D} 2$ \\
\hline \multirow[t]{2}{*}{ Fuji II LC (FII) } & \multirow[t]{2}{*}{$\mathrm{GC}$} & Powder: Aluminosilicate glass, pigments & A2 & $170526 \mathrm{~A}$ \\
\hline & & Liquid: Polyacrylic, hydroxymethyl methacrylate, water & B3 & $160806 \mathrm{~A}$ \\
\hline ChemFil Rock (CFR) & Dentsply & $\begin{array}{l}\text { Powder: Calcium-aluminium-zinc-fluoro-phosphor-silicate glass, } \\
\text { iron oxide, titanium dioxide pigments } \\
\text { Liquid: Polycarboxylic acid, tartaric acid, water }\end{array}$ & A2 & 1705000385 \\
\hline GC Fuji IX (FIX) & $\mathrm{GC}$ & $\begin{array}{l}\text { Powder: Fluoro-aluminosilicate glass, polyacrylic acid powder, } \\
\text { iron oxide, titanium dioxide } \\
\text { Liquid: Polyacrylic acid aqueous solution, tartaric acid, water }\end{array}$ & $\mathrm{A} 2$ & $170414 \mathrm{~A}$ \\
\hline IonoStar Plus (ISP) & Voco & $\begin{array}{l}\text { Powder: Fluoro-aluminosilicate glass } \\
\text { Liquid: Polyacrylic acid, (+)-tartaric acid }\end{array}$ & A2 & 1732397 \\
\hline Riva Self Cure (RSC) & SDI & $\begin{array}{l}\text { Powder: Fluoro-aluminosilicate glass } \\
\text { Liquid: Acrylic acid homopolymer, tartaric acid }\end{array}$ & A2 & B1606202F \\
\hline
\end{tabular}

placed into the standard cylindrical moulds described above. These were filled with the same material but in a different shade in order to help differentiate them from the base and clamped by glass slides. For FII, light curing was performed again following the same procedure above. Specimens were stored in the same water bath at $37^{\circ} \mathrm{C}$ for $1 \mathrm{~h}$, demoulded, transferred into glass jars and stored in the incubator (MIR262, Sanyo, Japan) at $37{ }^{\circ} \mathrm{C}$ for 1 day, 1 week, 1 month and 3 months.

All specimens were equally grouped according to the model and ageing time frame $(n=8)$.

\section{Compression test}

Compressive strength (CS) was measured using a universal testing machine (5569A, Instron, UK) fitted with a flat crosshead and a load cell (50 kN capacity). The extension speed of the crosshead was $1 \mathrm{~mm} / \mathrm{min}$. Specimens were kept moist prior to the test. Before each test, water was carefully blotted from the specimen surface. Specimens were placed on aluminium discs during the test. Compressive strength was calculated using the following equation:

$C S=\frac{P_{\max }}{S_{C S}}$

in which $P_{\max }$ denotes the maximum load and $S_{C S}$ denotes the contact area.

\section{Edge compression test}

Edge stability (ES) was evaluated by using the above compression test rig on the edge of the specimen using the same
Fig. 1 Schematics of preparation methods of standard (a) and repaired (b) models and respective standard cylindrical (c) and repaired (d) specimens. The convergence angle is $2.86^{\circ}$. Dimensions are in millimetres

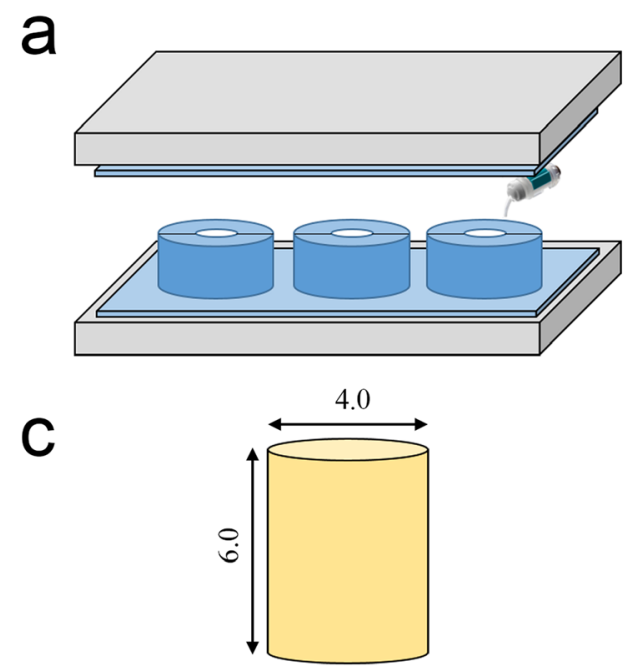

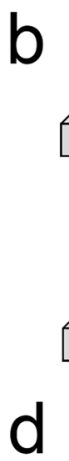
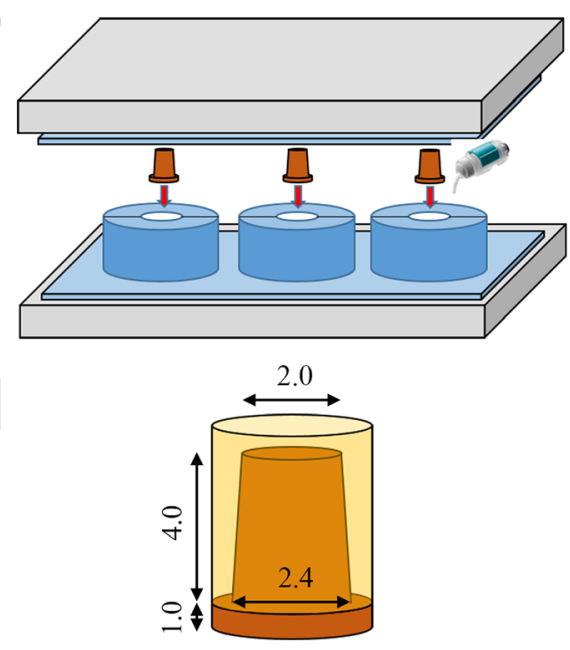
universal testing machine setup and parameters. A customized jig was utilized to ensure the position of the crosshead was on the edge of the specimen, as illustrated in Fig. 2a, b. The jig consisted of a metal cylinder with one flat end which contacts the crosshead and the other end which was fitted with a tip (round flat shape, diameter $2 \mathrm{~mm}$ ) contacting the specimen edge and a stage that allowed the cylinder to move vertically. Two long cylinders (supporters) on either side of the tip restricted the lateral movement of the specimen. To further ensure the stability, an open hoop was used to hold the specimen towards the supporters.

The contact area was calculated as illustrated in Fig. 2c. The following equation was used:

$S_{E S}=r^{2}\left(\cos ^{-1} \frac{7}{8}+\frac{1}{4} \sin ^{-1} \frac{1}{4}-\frac{\sqrt{15}}{8}\right)$

where $r$ denotes the radius of the specimen and the radius of the tip and the supporter is $1 / 2 r$ and $1 / 4 r$, respectively. In this study, the specimen had a diameter of $4 \mathrm{~mm}$; hence, the $r$ was $2 \mathrm{~mm}$, and $\mathrm{S}_{\mathrm{ES}}$ was calculated to be $1.42 \mathrm{~mm}^{2}$. In the edge compression test, only the maximum load was recorded. The edge stability was calculated as follows:

$E S=\frac{P_{\max }}{S_{E S}}$

in which $P_{\max }$ denotes the maximum load.

\section{Scanning electron microscopy}

After the mechanical tests, digital images of fractured specimens were captured by a digital camera (D800E, Nikon, Japan) equipped with a micro lens (Nikkor AF-S Micro $60 \mathrm{~mm} \mathrm{f} / 2.8 \mathrm{G}$ ED, Nikon, Japan). The specimens were sputter-coated with gold and analysed using a scanning electron microscope (JCM-6000 Plus, JEOL, Japan) in secondary electron mode with acceleration voltage of $15 \mathrm{kV}$.

\section{Statistical analysis}

All compressive strength and edge stability data were analysed statistically using one-way ANOVA with Tukey's test carried out in SPSS 23 for Windows (IBM, USA). Before ANOVA analysis, the normality and homogeneity of all data were checked by Shapiro-Wilk test and Levene test, respectively. Significance level was set at $p<0.05$.

\section{Results}

Figure 3 shows the compressive strength (mean \pm standard deviation) of all specimens in the standard model after the various ageing periods. KU exhibited the highest compressive strength among all materials at all time points $(p<0.05)$. In comparison, FII (RMGIC) showed the lowest. The strength of
Fig. 2 Illustrations of the set-up for the edge compression test: a the front view and $\mathbf{b} 45^{\circ}$ side view. The specimen was stabilized by two cylindrical supporters and an open hoop. The tip was positioned at the edge of the specimen. c shows the schematic of the contact area (shaded)

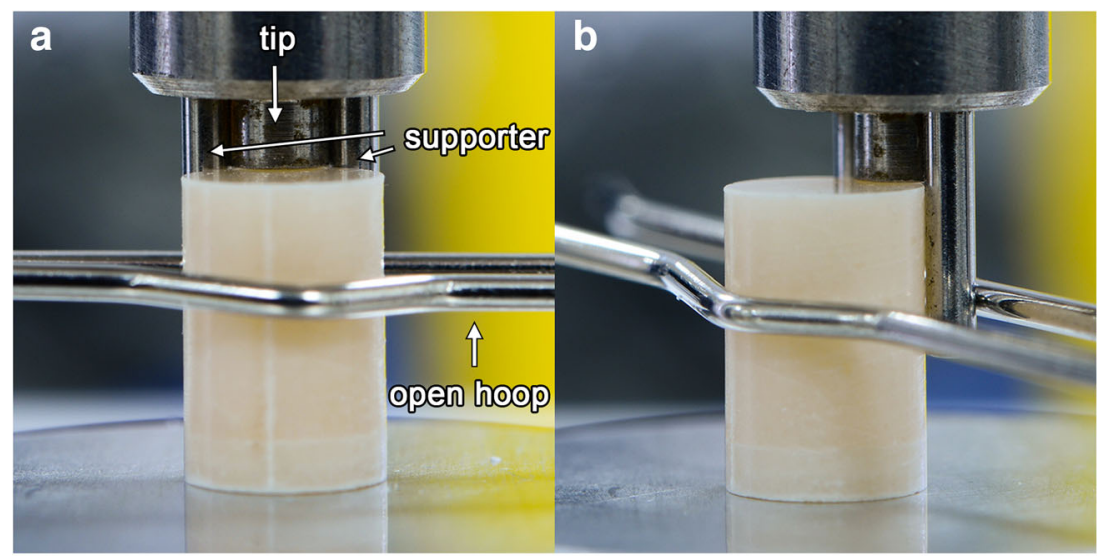

C

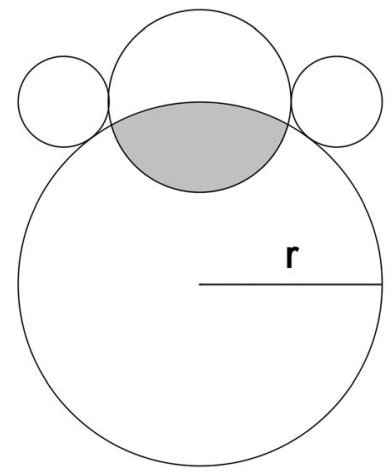




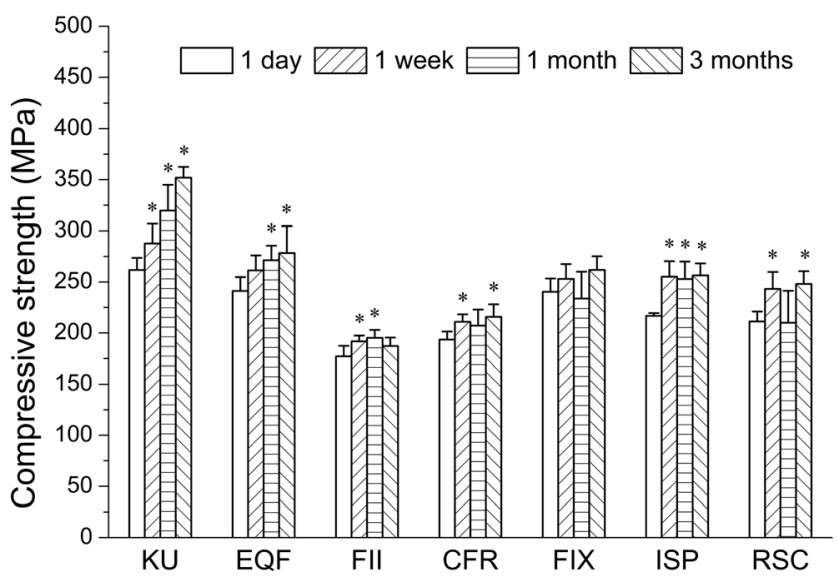

Fig. 3 Compressive strength of all standard specimens after different ageing times (mean \pm S.D., MPa). “*” indicates significant difference compared 1 day within each material $(p<0.05)$. KU showed greater compressive strength than other materials at all time points. The mean strength of KU reached $262 \pm 12 \mathrm{MPa}$ at 1 day and kept increasing with ageing. At 1 week, 1 month and 3 months, compressive strength of KU showed a significantly higher value compared to that at 1 day $(p<0.05)$. Other materials reached a plateau between 1 week and 1 month

KU significantly increased throughout the study from $262 \pm$ $12 \mathrm{MPa}$ at 1 day to $352 \pm 11 \mathrm{MPa}$ at 3 months $(p<0.05)$. For the other materials, the increase in compressive strength slowed at 1 week or 1 month time points. CFR, FIX, ISP and RSC exhibited a decrease in CS at 1 month.

The edge stability of all standard specimens is shown in Fig. 4. A similar tendency as in Fig. 3 was found with KU showing better edge stability than others at all time points except 1 day. In addition, the edge stability of KU grew after 1 week and 1 month, which was significantly greater than that after 1 day $(p<0.05)$. After 1 month, other materials started to show stability, but a reduction of stability was found for KU

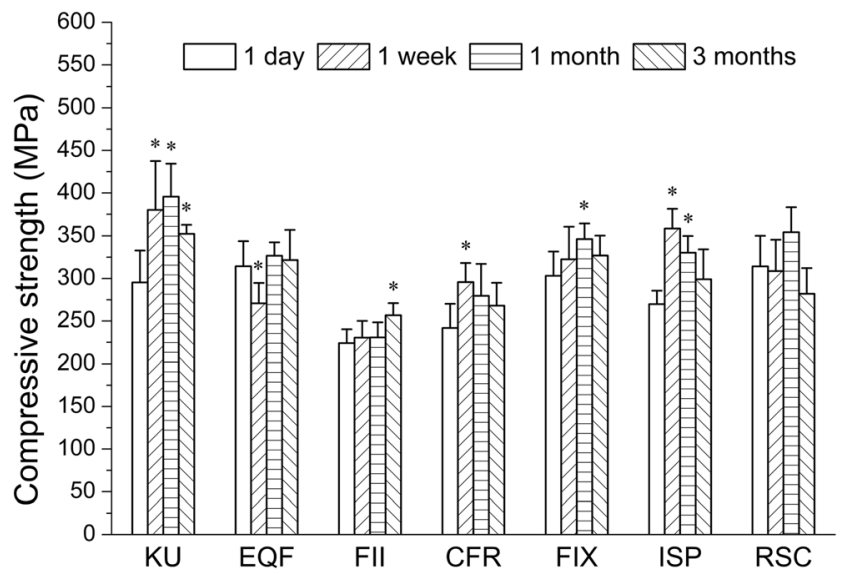

Fig. 4 Edge stability of all standard specimens after different ageing times (mean \pm S.D., MPa). "*” indicates significant difference compared 1 day within each material $(p<0.05)$. Although KU exhibited a lower value $(295 \pm 38 \mathrm{MPa})$ than EQF $(314 \pm 30 \mathrm{MPa})$ at 1 day, no statistical difference was found between these groups. Edge stability of KU significantly increased after 1 week and exceeded those of other groups, then showed a reduction at 3 months

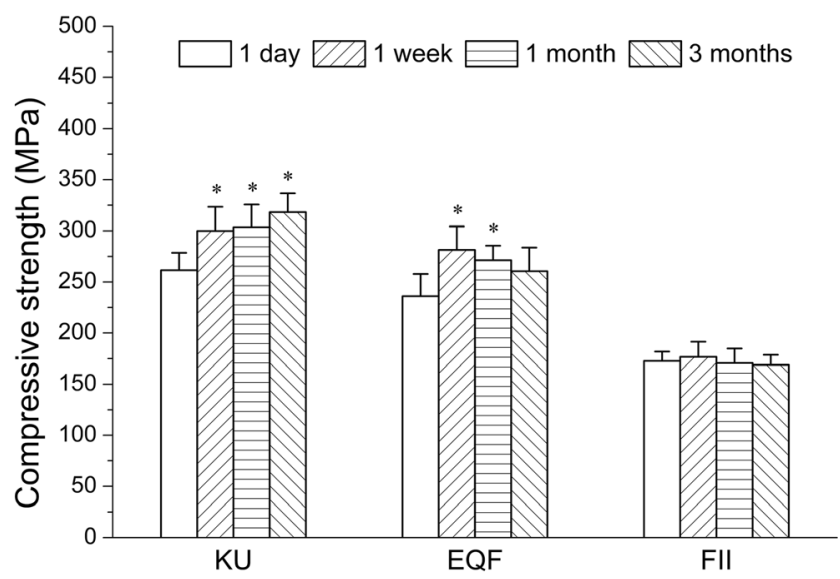

Fig. 5 Compressive strength of all repaired specimens after different ageing times (mean \pm S.D., MPa). “*” indicates significant difference compared 1 day within each material $(p<0.05)$. The compressive strength of the repaired KU significantly increased after 1-week ageing $(p<0.005)$, and then reached a plateau. In EQF, a decreasing trend was noticed, despite no statistical differences being observed $(p>0.05)$. In FII, ES showed no difference at all time points $(p>0.05)$

and RSC at 3 months. By comparing Fig. 4 to Fig. 3, it was noticed that all specimens had a greater value in edge stability than that in compressive strength at the corresponding time points. However, the standard deviation was also higher in the edge stability tests, especially at 1 day and 1 week time points.

For the repair model, the compressive strength and the edge stability are summarized in Figs. 5 and 6, respectively. Again, KU showed greater compressive strength than other materials (statistically significant except at 1 week). At 1 day and 1 week, the strength of the repaired KU $(262 \pm 17$ and $300 \pm$ $24 \mathrm{MPa}$, respectively) was similar to that of the standard KU (262 \pm 12 and $288 \pm 19 \mathrm{MPa}$, respectively), whilst at 1 month and 3 months $304 \pm 22$ and $318 \pm 18 \mathrm{MPa}$, respectively), it was significantly lower than the standard values $(320 \pm 25$

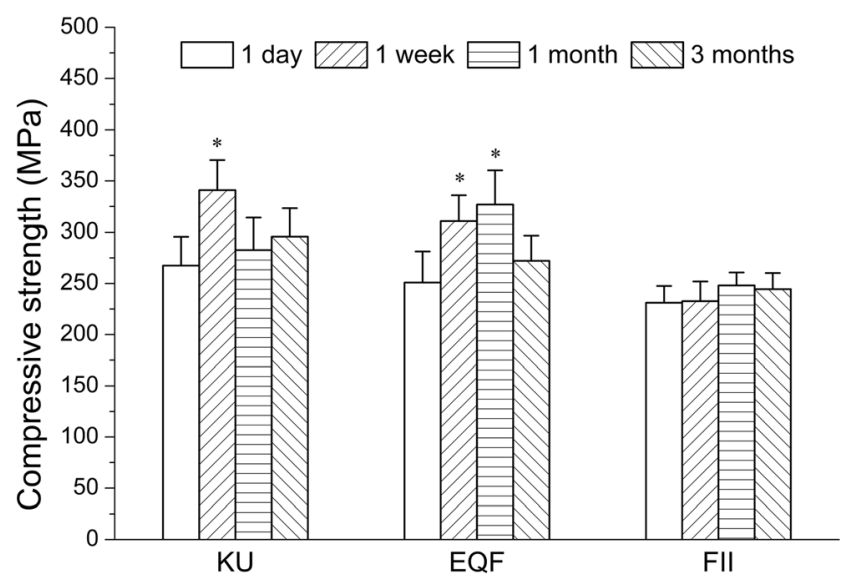

Fig. 6 Edge stability of all repaired specimens after different ageing times (mean \pm S.D., MPa). “*” indicates significant difference compared 1 day within each material $(p<0.05)$. Edge stability of the repaired KU reached the maximum value of $341 \pm 29$ after 1 week, followed by a decrease. EQF showed a similar trend except the decrease appeared after 1 month. No difference was found in FII $(p>0.05)$ 
and $352 \pm 11 \mathrm{MPa}$, respectively). A similar tendency was also found in the repaired EQF and FII specimens. With respect to the edge stability of the repaired specimens, all three materials showed a weaker stability at 1 day than the corresponding values shown in Fig. 4. Despite the increase after 1-week ageing, the stability $(341 \pm 29 \mathrm{MPa})$ was still weaker than that in Fig. $4(380 \pm 57 \mathrm{MPa})$, which was followed by a significant reduction after 1 month $(296 \pm 28 \mathrm{MPa})(p<0.05)$. For the repaired EQF, the edge stability kept increasing until 1 month. Regarding the repaired FII, the edge stability was generally stable throughout the entire time frame and the values were close to those in Fig. 4.
Fractured pieces of standard KU specimens after compression tests showed a typical pyramidal shape at all time points, as shown in Fig. 7a-d, whereas in FII group, failed specimens maintained the bulk structure despite cracking (Fig. 7i-1). Specimens from all the other groups also showed shape after tests similar to that in KU (see Figs. S1 to S5). The edge compression led to a different behaviour that not only the edge of the tested surface was chipped away, but also there were cracks propagating along the diagonal direction (arrows, Fig. 7e, $\mathrm{g}, \mathrm{h}$ ). Some specimens showed a diagonal loss of the material at 1 week (Fig. 7f), which was also observed in other groups (see Figs. S1 to S5). FII showed similar fracture after edge
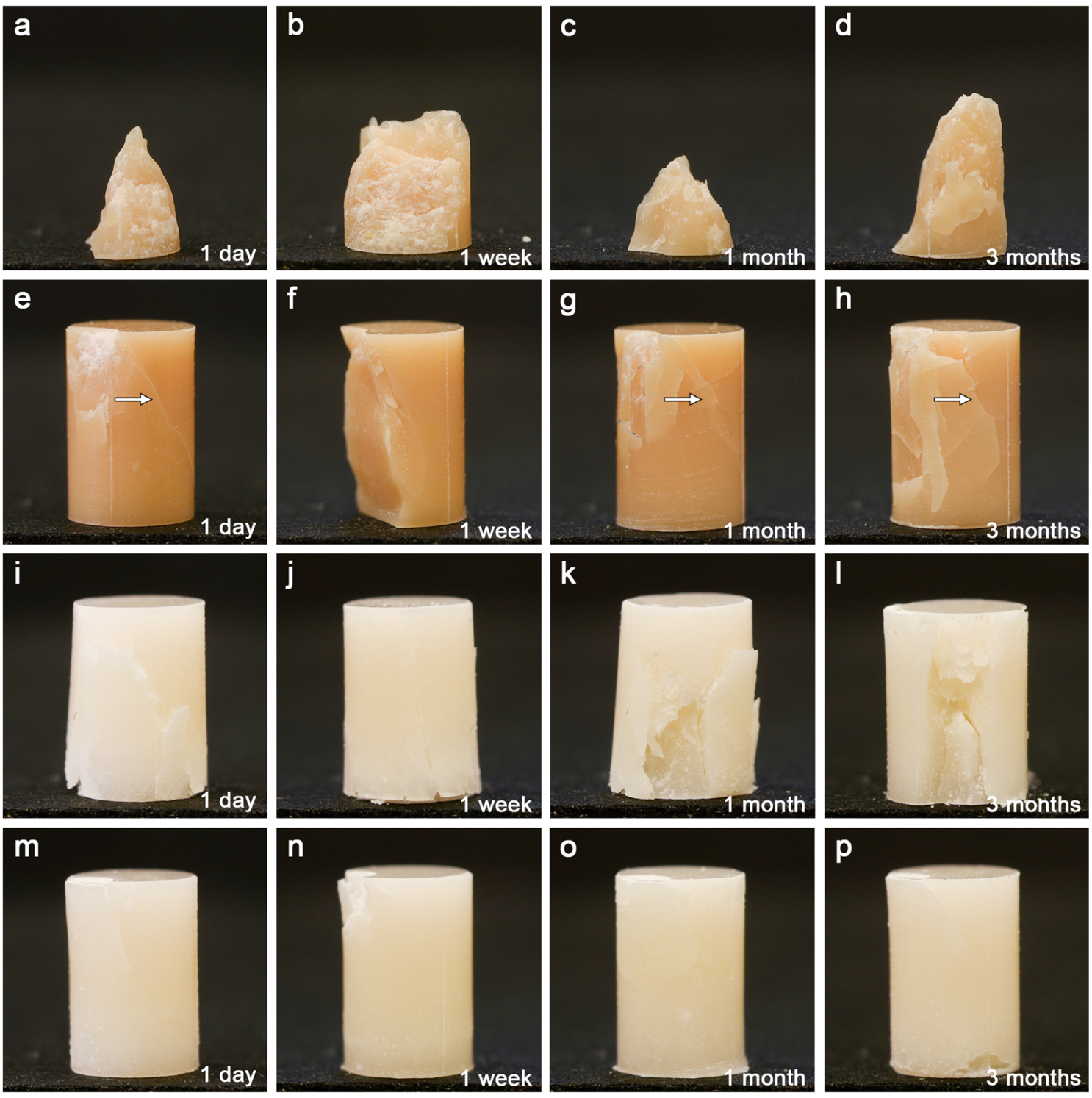

Fig. 7 Digital photos of the representative standard KU (a-d) and FII specimens (i-l) after compression tests and edge tests (KU: $\mathbf{e}-\mathbf{h} ;$ FII: $\mathbf{m}-$ p). Typical pyramid shape was observed for all standard KU specimens after compression tests. At 1 week, KU exhibited a break along the diagonal direction from the contact area (f) after edge compression. Other specimens did not show the diagonal loss, but cracks were clearly
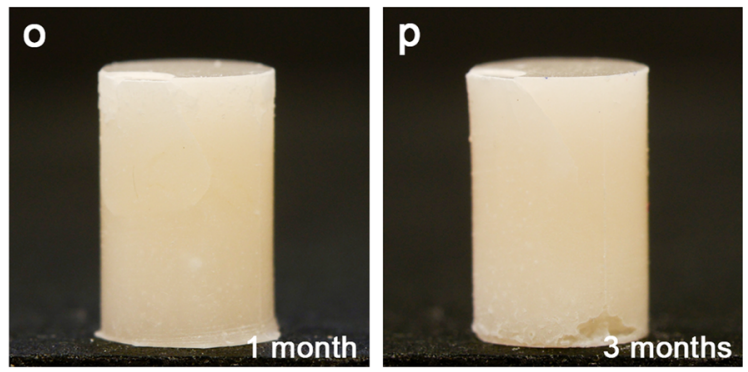

seen in this direction (arrows in $\mathbf{e}, \mathbf{g}, \mathbf{h}$ ). FII specimens maintained the bulk structure after compression tests with some cracks ( $\mathbf{i}-\mathbf{j})$ after 1 day and 1 week, and after 1 month, the structure appeared to collapse after tests $(\mathbf{k}, \mathbf{l})$. No diagonal loss was found for FII after edge tests, although diagonal cracks were present $(\mathbf{m}-\mathbf{p})$ 
compression; however, no diagonal loss but only cracks were observed (Fig. 7m-p). As a comparison, Fig. 8 illustrated the broken pieces of the repaired KU and FII specimens. At 1 day, compression led to a complete delamination of the repairing material from the base in KU as shown in Fig. 8a. However, with longer ageing, broken pieces exhibited the pyramidal shape as those in Fig. 7. Edge compression induced diagonal loss and this was observed in all specimens (Fig. 8e-h). The fracture passed through both the repair material and the base. Repaired EQF showed broken pieces similar to KU (Fig. S6). The repair of FII did not result in significant changes to the fracture after both tests compared to standard FII specimens (Fig. 8i-p).
SEM micrographs show the ultrastructure of the repaired $\mathrm{KU}$ and $\mathrm{EQF}$ at 1 week and 1 month after the edge compression (Fig. 9). The base had a screw thread pattern which came from the in-wall of the mould. Obvious gaps were recognized at the interfaces between the repair material and the base for both materials (Fig. 9a, c). After 1-week ageing, however, it seems that the bonding at the interface was improved as no obvious gaps were observed (Fig. 9c, d). SEM observations could not be carried out on the repaired FII specimens because no interface or cross-sectioned surfaces were exposed after the edge testing failures.
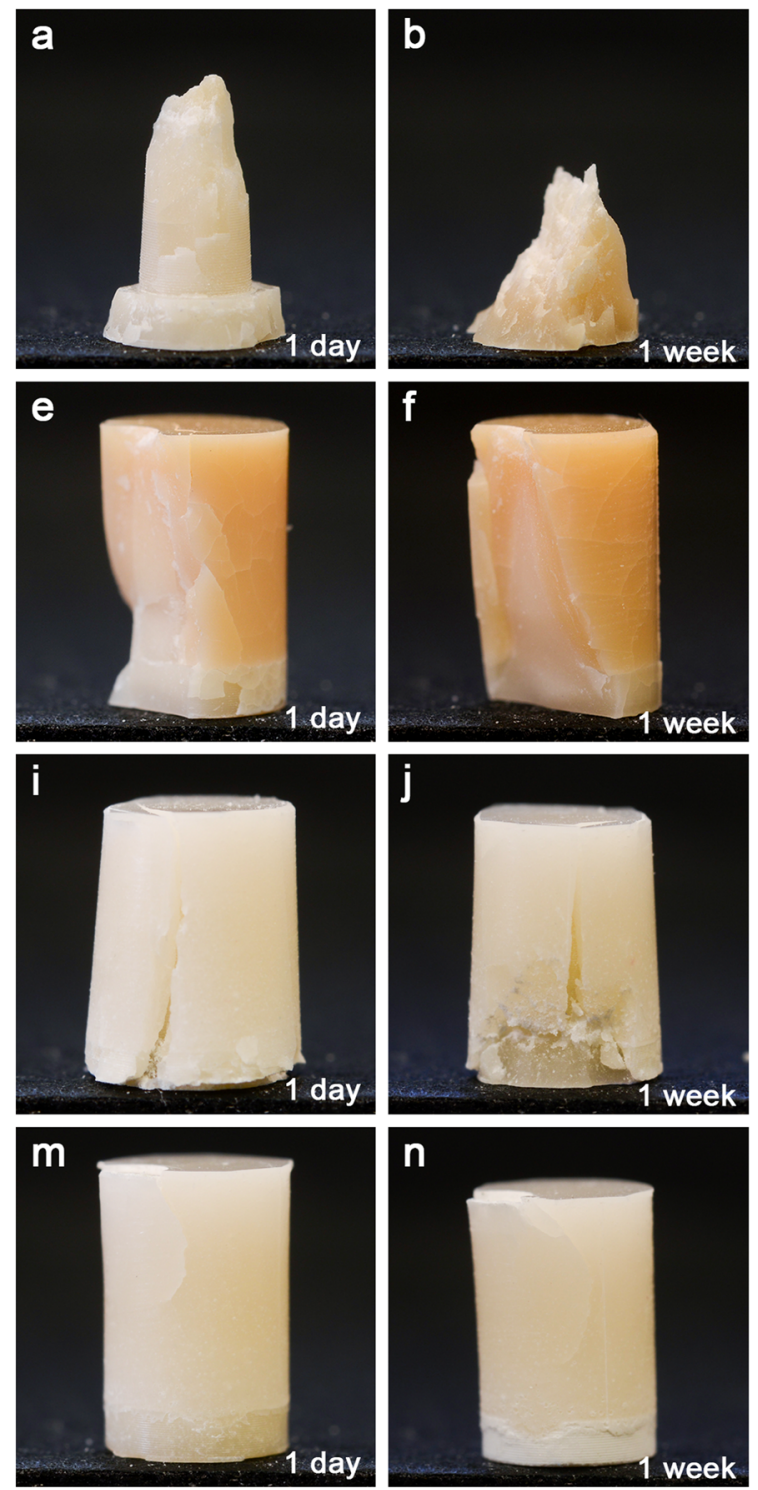

Fig. 8 Digital photos of the representative repaired KU and FII specimens after compression (a-d and $\mathbf{i}-\mathbf{l}$, respectively) and edge (e-h and $\mathbf{m}-\mathbf{p}$, respectively) tests. At 1 day, the cone-shape base (a) was exposed with an almost entire structure after compression test, while at other time points, typical pyramid shape pieces were observed (b-d). After
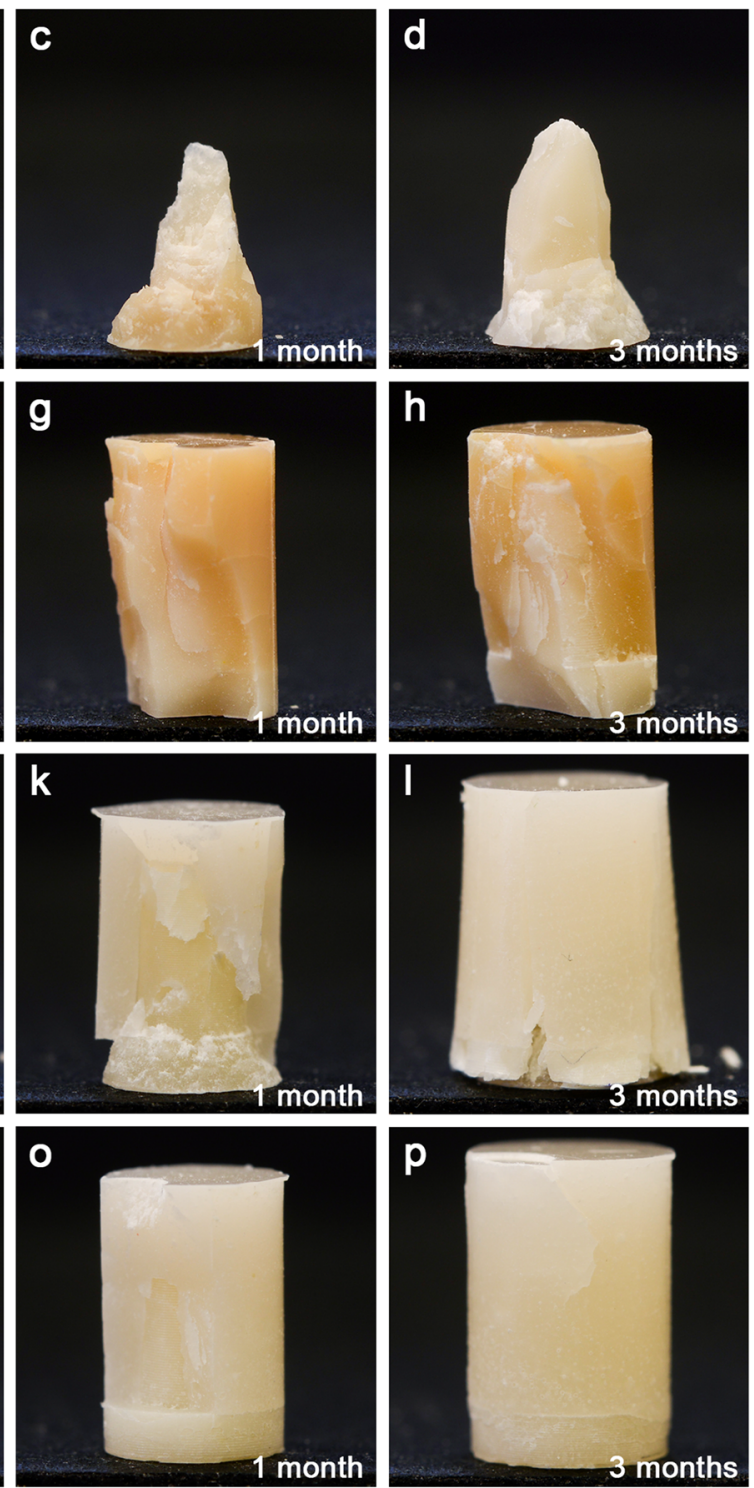

edge compression, all specimens revealed a diagonal loss of the material, and the break went through both base and repairing materials $(\mathbf{e}-\mathbf{h})$. Repaired FII specimens did not show complete fracture after compression tests; however, delamination seemed to have occurred (i-l). Edge tests led to cracks along the diagonal direction $(\mathbf{m}-\mathbf{p})$ 


\section{Discussion}

In clinical studies, marginal restoration fracture was observed frequently as one of the primary reasons for GIC failure [9]. Studies investigating the edge toughness (stability) have long been neglected. Quinn et al. $[10,11]$ reported a series of edge chipping studies by using a commercial chipping machine. Their findings suggest that comparisons could be made between materials, although the results may vary due to a number of reasons including the type of indenter and the size of tested area. More recently, an edging testing method was described by Ilie [12]. This method featured a bespoke jig that allows specific marginal assessment to be made on the edge $\left(\sim 1.4 \mathrm{~mm}^{2}\right)$. In the present study, this edge testing methodology was adapted to incorporate more lateral stability of the samples using an open hoop. It was used on all normal and repaired specimens of all groups and the data were compared with conventional compression testing.

In comparison to conventional compression strength testing, edge stability results showed greater mean values at all time points. The explanation behind this is unclear. It is accepted that the fracture of a material is the result of crack propagation. In this study, the contact area during edge testing was $1.42 \mathrm{~mm}^{2}$, indicating only a small volume of material withstood the force, which can also be seen from the fractured pieces (Fig. 7). Therefore, perhaps fewer voids propagated until failure. More studies are necessary to explain this behaviour. Nonetheless, it was also found that repaired GICs (KU, EQF and FII) had similar edge stability to their corresponding normal specimens after ageing in water until 1 month, except for repaired KU in which ES dropped after 1-month immersion. In addition, the fact that ES was greater than CS differs from Ilie's results [12]. This might be due to the jig design in which an open hoop was used in the present study to limit the side movement of specimen during test. It was also noted that FII showed the weakest results compared to the other materials in almost all tests. This could be due to its higher water take-up during the storage period. Cattani-Lorente et al. [23] found that Fuji II LC had a surface sensitive to water. In resinmodified glass-ionomer cements, acid-base reactions take place along with the polymerization reaction, resulting in a complex structure consisting of ionic and covalent crosslinks. The water content may not only contribute to the volumetric expansion but more importantly cause phase separation, which would eventually deteriorate its mechanical properties.

Historical research has been carried out on repairing failed glass-ionomer cement restorations using a conventional cylindrical in-vitro model [18, 20, 24]. Seldom have investigations been accomplished on conventional GIC rather than RMGIC. In a previous study, surface conditioning was applied before repairing using the same material and phosphoric acid etching offered the best repair [20]. In this study, an in vitro repair model was proposed. Conventional compression test results suggest that the repaired specimens, regardless of GIC type, showed overall similar compressive strengths across the entire period. Cattani-Lorente et al. [25] investigated the influence of water-storage on GICs' mechanical performance and concluded that additional crosslinking and build-up of a silica gel phase might account for the increase in mechanical strength. This is in agreement with current results. However, a clear gap was found between the repaired layer and the base in KU after 1-day ageing (Fig. 9) whereas compressive strength still reached the value of normal specimens (Figs. 3 and 5). This may imply that imperfections in the GICs at their early maturation stage play a role during the collapse of structure. It was noticed that the repaired RMGIC (Fuji II LC) showed a
Fig. 9 SEM micrographs (magnification $\times 40$ ) of the repaired $\mathrm{KU}$ and $\mathrm{EQF}$ specimens aged for 1 day $(\mathbf{a}, \mathbf{c})$ and 1 week (b, d) after edge compression tests. Clear gap at the interface was observed at 1 day for both group $(\mathbf{a}, \mathbf{c})$. The screw thread pattern was caused by the mould. At 1 week, although there was delamination at the bottom of the image of repaired $\mathrm{KU}$, no extension was found (b), which is similar to that in EQF except no obvious interface was observed (d). The dash line in $\mathbf{b}$ indicates the interface between the base and the repairing $\mathrm{KU}$ material
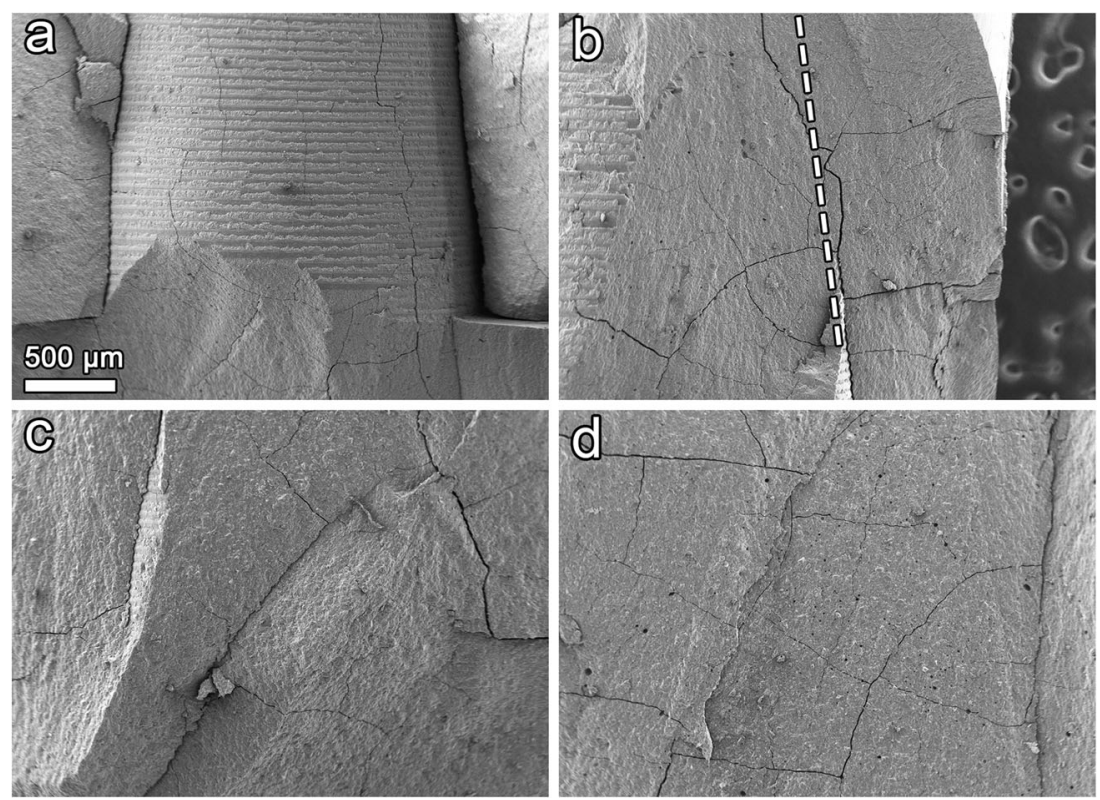
relatively stable compressive strength, and the values were similar to those in the normal specimens. This contradicts the findings of Maneenut et al. [21] who concluded that repair of RMGIC to RMGIC was unpredictable. Edge stability of the repaired KU samples started to decrease after 1-month ageing in water. It was also noted that after ageing for 3 months immersion, repaired specimens showed weaker mechanical properties on both CS and ES tests compared to the standard specimens. This is in line with Yap et al. [24]. Several factors may account for the relative weakening. For example, water, resident within the interface between the repaired layer and the base could reduce the mechanical performance. Erosion and plasticising effects of water may cause weakening in GIC prepared in a normal mould [25].

In the present study, an in vitro model for direct repair was deployed, which attempts to represent a more clinically relevant scenario. This is a simplified in vitro model comprising a cone-shaped base. The rationale behind this shape was to design a simple non-split mould which is easy to reproduce. The convergence angle was $2.86^{\circ}$, which follows the principle of Total Occlusal Convergence that for the ideal crown preparation, the total taper of the axial walls of the preparation should not exceed 6 to $8^{\circ}[26]$. Another consideration was the ease of demoulding the material. This design minimizes the opportunities of surface imperfections being introduced which would lead to incomplete condensation of the tested materials from taking place during demoulding. However, in clinical practice, situations are usually rather complex because the shape of the failed TRC varies. The irregular shape may result in imperfections between the old and new GIC materials. Additionally, despite statistical differences being noted during the comparison tests, the real-life clinical difference might not be significant in this study. The purpose of this study is an initial validation of a novel in vitro restoration repair model. In order to observe more clinical relevance, further studies are needed to improve this model by altering the shape or introducing imperfections on the repair base model.

\section{Conclusions}

In the present study, a novel GIC in vitro repair model consisting of a cone-shaped base and a repaired layer has been introduced. The mechanical properties were evaluated by conventional compression test and an adapted edge compression test. Repaired GIC had compressive strength and edge stability similar to normal GIC specimens after ageing in water for 1 week and 1 month. Therefore, the null hypotheses were rejected. Under current experimental conditions, GICs could be repaired by directly applying the same materials on the remaining broken pieces for a short duration with compressive strength and edge stability similar to standard GICs.
Acknowledgements The authors would like to thank the late Mr. Richard Mallet and Mr. Peter Pilecki for their assistance in the mechanical testing laboratory procedures.

Funding information This independent researcher-led study was supported financially by a grant from $3 \mathrm{M}$.

\section{Compliance with ethical standards}

Conflict of interest Peter Braun is employed by 3M. Jing Zhang and Avijit Banerjee declare that they have no conflict of interest.

Ethical approval This article does not contain any studies with human participants or animals performed by any of the authors.

Informed consent For this type of study, formal consent is not required.

Open Access This article is licensed under a Creative Commons Attribution 4.0 International License, which permits use, sharing, adaptation, distribution and reproduction in any medium or format, as long as you give appropriate credit to the original author(s) and the source, provide a link to the Creative Commons licence, and indicate if changes were made. The images or other third party material in this article are included in the article's Creative Commons licence, unless indicated otherwise in a credit line to the material. If material is not included in the article's Creative Commons licence and your intended use is not permitted by statutory regulation or exceeds the permitted use, you will need to obtain permission directly from the copyright holder. To view a copy of this licence, visit http://creativecommons.org/licenses/by/4.0/.

\section{References}

1. Anusavice K (1996) Challenges to the development of esthetic alternatives to dental amalgam in an academic research center. Trans Acad Dent Mater 83:25-50

2. Yip HK, Tay FR, Ngo H, Smales RJ, Pashley DH (2001) Bonding of contemporary glass ionomer cements to dentin. Dent Mater 17: 456-470

3. Berg JH (2002) Glass ionomer cements. Pediatr Dent 24:430-438

4. Banerjee A, Watson TF (2015) Pickard's guide to minimally invasive operative dentistry, 10th edn. Oxford University Press, Oxford

5. Meyer JM, Cattani-Lorente MA, Dupuis V (1998) Compomers: between glass-ionomer cements and composites. Biomaterials 19: 529-539

6. Köhler B, Rasmusson CG, Ödman P (2000) A five-year clinical evaluation of class II composite resin restorations. J Dent 28:111116

7. Burke FJT, Wilson NHF, Cheung SW, Mjör IA (2001) Influence of patient factors on age of restorations at failure and reasons for their placement and replacement. J Dent 29:317-324

8. Frankenberger R, Garcia-Godoy F, Lohbauer U, Petschelt A, Krämer N (2005) Evaluation of resin composite materials. Part I: in vitro investigations. Am J Dent 18:23-27

9. Ruengrungsom C, Palamara JEA, Burrow MF (2018) Comparison of ART and conventional techniques on clinical performance of glass-ionomer cement restorations in load bearing areas of permanent and primary dentitions: a systematic review. J Dent 78:1-21

10. Quinn GD, Giuseppetti AA, Hoffman KH (2014) Chipping fracture resistance of denture tooth materials. Dent Mater 30:545-553 
11. Quinn GD, Giuseppetti AA, Hoffman KH (2014) Chipping fracture resistance of dental CAD/CAM restorative materials: part I - procedures and results. Dent Mater 30:e99-e111

12. Ilie N (2018) Maturation of restorative glass ionomers with simplified application procedure. J Dent 79:46-52

13. Green DJ, Mackenzie L, Banerjee A (2015) Minimally invasive long-term management of direct restorations: the "5Rs". Dent Update 42:413-426

14. Setcos JC, Khosravi R, Wilson NH, Shen C, Yang M, Mjör IA (2004) Repair or replacement of amalgam restorations: decisions at a USA and a UK dental school. Oper Dent 29:392-397

15. Blum IR, Jagger DC, Wilson NH (2011) Defective dental restorations: to repair or not to repair? Part 1: direct composite restorations. Dent Update 38:78-84

16. Martin J, Fernandez E, Estay J, Gordan VV, Mjör IA, Moncada G (2013) Management of Class I and Class II amalgam restorations with localized defects: five-year results. Int J Dent 450260

17. Hickel R, Brüshaver K, Ilie N (2013) Repair of restorations criteria for decision making and clinical recommendations. Dent Mater 29:28-50

18. Parra M, Kopel HM (1992) Shear bond strength of repaired glass ionomers. Am J Dent 5:133-136

19. Pearson GJ, Bowen G, Jacobsen P, Atkinson AS (1989) The flexural strength of repaired glass ionomer cements. Dent Mater 5:10 12
20. Jamaluddin A, Pearson GJ (1994) Repair of glass ionomer cements - methods for conditioning the surface of the cement to achieve bonding. J Oral Rehabil 21:649-653

21. Maneenut C, Sakoolnamarka R, Tyas MJ (2010) The repair potential of resin-modified glass-ionomer cements. Dent Mater 26:659665

22. Welch D, Seesengood B, Hopp C (2015) Surface treatments that demonstrate a significant positive effect on the shear bond strength of repaired resin-modified glass ionomer. Oper Dent 40:403-409

23. Cattani-Lorente MA, Dupuis V, Moya F, Payan K, Meyer JM (1999) Comparative study of the physical properties of a polyacid-modified composite resin and a resin-modified glass ionomer cement. Dent Mater 15:21-32

24. Yap AUJ, Lye KW, Sau CW (2000) Effects of aging on repair of resin-modified glass-ionomer cements. J Oral Rehabil 27:422-427

25. Cattani-Lorente MA, Godin C, Meyer JM (1994) Mechanical behavior of glass ionomer cements affected by long-term storage in water. Dent Mater 10:37-44

26. Mamoun JS (2013) The total occlusal convergence of the abutment of a partial fixed dental prosthesis: a definition and a clinical technique for its assessment. Eur J Dent 7:509-513

Publisher's note Springer Nature remains neutral with regard to jurisdictional claims in published maps and institutional affiliations. 